\title{
Nyquist-WDM With Low-Complexity Joint Matched Filtering and Adaptive Equalization
}

\author{
Chen Zhu, Student Member, IEEE, Bill Corcoran, An V. Tran, Senior Member, IEEE, \\ and Arthur J. Lowery, Fellow, IEEE
}

\begin{abstract}
In this letter, we investigate the application of jointly processing the matched filtering and blind adaptive equalization functions in a receiver for a Nyquist wavelength division multiplexing (Nyquist-WDM) system employing a root-raised-cosine (RRC) pulse shaping filter. We show that blind equalization is able to also provide the matched filtering function, if the number of filter taps is increased. In addition, if implemented in the frequency domain, this form of blind equalization is more computationally efficient than when a separate RRC is used. It is also verified that this method is not sensitive to frequency offset. We demonstrate a 16-channel 19-GBd Nyquist-WDM system with QPSK and 16-quadrature-amplitude-modulation formats, and find the frequency domain blind equalizer can replace the RRC filter without adding a performance penalty.
\end{abstract}

Index Terms-Coherent optical system, matched filtering, blind adaptive equalization, QPSK, 16-QAM.

\section{INTRODUCTION}

$\mathbf{T}$ O COPE with the ever increasing demand for data transmission, the bit rate per wavelength in high-speed optical systems is likely to increase from $100 \mathrm{~Gb} / \mathrm{s}$ to $400 \mathrm{~Gb} / \mathrm{s}$ or even $1 \mathrm{~Tb} / \mathrm{s}$ [1]. Improving spectral efficiency plays a significant role in supporting such a high increase. Besides the combination of polarization-division-multiplexing (PDM) and high-order quadrature-amplitude-modulation (QAM) formats, there is a growing interest in using digital Nyquist pulse shaping to generate near-rectangular spectra to reduce the WDM channel spacing to towards the symbol rate of each channel [2]-[5].

Raised-cosine (RC) pulses are usually chosen to satisfy the Nyquist theorem [6]. In such systems, matched filtering can be exploited to provide the best performance [7], by implementing root-raised-cosine (RRC) filters at both the transmitter and receiver, to realize an overall RC spectral shape. RRC filters with a small roll-off factors lead to near-rectangular spectra, and therefore enable closer channel spacings, at the cost of

Manuscript received July 25, 2014; revised September 1, 2014; accepted September 2, 2014. Date of publication September 8, 2014; date of current version October 28, 2014. This work was supported by the Australian Research Committee Laureate Fellowship under Project FL130100041.

C. Zhu is with the Department of Electrical and Computer Systems Engineering, Monash University, Clayton, VIC 3800, Australia (e-mail: chen.zhu@monash.du).

B. Corcoran and A. J. Lowery are with the Department of Electrical and Computer Systems Engineering, Monash University, Clayton, VIC 3800, Australia, and also with the Centre for Ultrahigh Bandwidth Devices for Optical Systems, University of Sydney, NSW 2006, Australia (e-mail: bill.corcoran@monash.edu; arthur.lowery@monash.edu).

A. V. Tran is with the Centre for Technology Infusion, La Trobe University at Bundoora, Melbourne, VIC 3086, Australia (e-mail: an.tran@latrobe.edu.au).

Color versions of one or more of the figures in this letter are available online at http://ieeexplore.ieee.org.

Digital Object Identifier 10.1109/LPT.2014.2355203
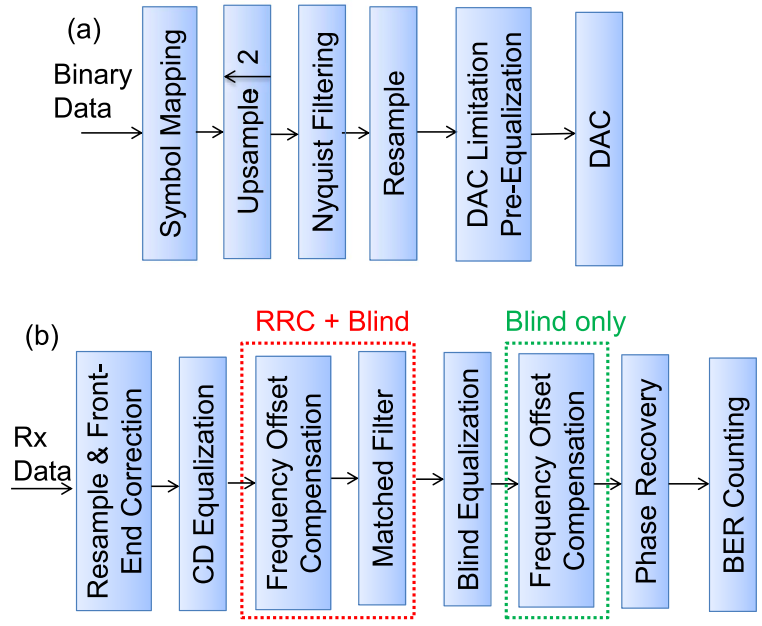

Fig. 1. (a) Transmitter and (b) receiver DSP flow for Nyquist-WDM system.

increased number of filter taps. A RRC filter with a very small roll-off factor $(\alpha \sim 0.01)$ may require a few hundreds of taps to implement; thus the receiver RRC filter and CD equalizer can be combined to reduce the computational cost [8]; however this demonstration was sensitive to frequency offset which causes performance degradation.

In this letter, we solve this issue by integrating the matched filter function into the conventional blind equalizer. We extend our previous work [9], to include a complete theoretical analysis and additional QPSK experimental results. We first show that accurate frequency offset compensation is necessary when implementing a receiver-side RRC filter. We then show that a blind equalizer with an increased number of taps is able to converge to an optimal linear solution that combines matched filtering and polarization de-multiplexing. We show that the blind equalizer is insensitive to frequency offset and that separate processing for the receiver's matched filtering can be omitted, at the cost of more taps. It is also demonstrated that a frequency domain implementation of such a longtap adaptive equalizer leads to a huge computational advantage. We conduct a 16-channel Nyquist WDM experiment with both PDM-QPSK and PDM-16-QAM modulation, and compare the system performance with and without separate matched filtering. This shows that a blind equalizer with frequency domain implementation leads to a computationally efficient solution for receiver processing in Nyquist WDM systems.

\section{System Digital Signal Processing}

\section{A. Transmitter's DSP Procedure}

Figure 1(a) illustrates the transmitter-side digital signal processing (DSP). The data bits are first mapped into symbols 
with either QPSK or 16-QAM modulation, then the signals are up-sampled to 2 samples/symbol, then fed to a near-ideal Nyquist-pulse-shaping RRC filter with a 0.01 roll-off factor, which is implemented in frequency domain with 256 taps. To generate a high baud rate signal with a limited DAC sample rate, the signals are resampled to 1.31 samples/symbol (1.52 down-sample factor from 2 to 1.31). Finally, a preemphasis filter is used to overcome the frequency roll-off of the digital to analog converter (DAC) and optical I/Q modulator.

\section{B. Receiver's DSP Procedure}

The receiver offline DSP algorithms are shown in Fig. 1(b). After Gram-Schmidt orthogonalization front-end impairment correction [10], the received signals are resampled to 2 samples/symbol. The chromatic dispersion (CD) is first compensated using an overlapsave frequency domain equalizer [11]. For the reference system that uses RRC matched filtering plus blind equalization $(\mathrm{RRC}+$ blind $)$, frequency offset compensation (FOC) is required before the RRC filtering; for our proposed system without separate RRC filtering (blind equalization only), the FOC is performed after the blind equalizer. A blind adaptive equalizer with constant modulus algorithm and a dual-mode algorithm [12] are used to remove residual linear impairments for the QPSK and 16-QAM modulation formats, respectively. For QPSK, standard differential Viterbi-Viterbi and Viterbi-Viterbi fourth power law are applied for frequency offset and phase noise estimation, respectively [10]. While for 16-QAM, the frequency offset and phase noise estimation is based on the digital decision-directed phase locked loop [13].

For the reference $(\mathrm{RRC}+$ blind) scheme, an accurate FOC function is necessary because the RRC matched filter is essentially a low-pass filter; if it is directly applied to the received base band signal which is frequency shifted due to frequency offset, the signal spectrum would be narrowed and therefore leads to performance degradation. Typically the frequency offset can be compensated effectively when all linear impairments are removed, i.e. FOC is usually placed after the $\mathrm{CD}$ and blind equalizer [14]. Therefore compensating the frequency offset before the matched filter and blind equalization is difficult and may require extra training sequences [15], also residual frequency offset may exist due to the uncompensated polarization crosstalk.

Alternatively, we can enlarge the filter size of the blind equalizer, to take care of the extra inter symbol interference introduced by the transmitter side digital pulse shaping. In this case, a separate matched filter is not necessary and therefore a conventional FOC module can be utilized after blind equalization. Intuitively, one might assume that a modest increase in the number of taps in the blind equalizer (no more than 30 taps as shown in later experiment) may offset the requirement for long tap RRC (256 tap for 0.01 roll-off RRC filter), reducing computational complexity. However, the computational cost may change in the opposite way, as one adaptive equalizer tap is more computationally expensive than one RRC filter tap. This is because: $i$ ) the adaptive equalizer is a multiple input multiple output (MIMO) filter while the RRC filter is a single input single output filter; ii) the adaptive equalizer needs to update the filter coefficients at every iteration, while the RRC filter coefficients are fixed. Thus, a frequency domain

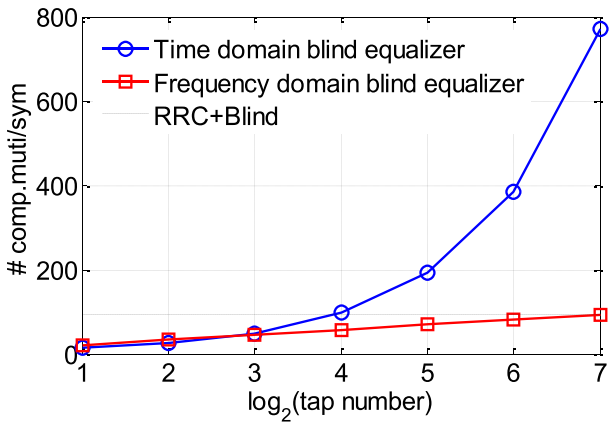

Fig. 2. Computational complexity comparison between time domain and frequency domain adaptive equalizer without matched filtering, the black curve includes a 256-tap RRC and 16-tap frequency domain blind equalizer is used as a reference.

implementation of such a long-tap equalizer [12], [16] could be of benefit.

The computational complexity comparison in terms of complex multiplications per symbol (comp.multi/sym) between time domain and frequency domain adaptive equalization is shown in Fig. 2 [16]. The reference black curve is calculated based on a 256-tap RRC filter and a 16-tap MIMO blind equalizer both implemented in the frequency domain with $50 \%$ overlap The RRC filter requires one 256-tap FFT/IFFT pair and 256 coefficient multiplications to output every 64 symbols. It is clear that when a time domain blind equalizer is used, the required computational cost is the highest when the number of taps number exceeds 16, otherwise the frequency domain implementation is the most computational-efficient solution. As such, our proposed method is beneficial if the frequency domain equalizer has between 16 to 128 taps, and provides a similar system performance to the RRC + blind equalizer.

\section{EXPERIMENTAL DEMONSTRATION}

The experimental setup is shown in Fig. 2. There were 8 external cavity lasers (ECLs) with $40-\mathrm{GHz}$ spacing in the transmitter (inset (i) of Fig. 3); these carriers were polarization controlled then power equalized by a Waveshaper (WS). A 25-Gsamples/s arbitrary waveform generator (AWG) was employed to generate the 19-GBaud baseband signals (giving a rational oversampling factor in the transmitter side DSP). Analogue electrical low-pass filters are used to remove the spurious frequency components of the AWG output. The RF signals were then amplified and used to drive an optical I/Q modulator, producing a half-filled spectrum (inset (ii) of Fig. 3). The optical signal was then split into two paths with a 50:50 coupler. One path was frequency shifted by $20 \mathrm{GHz}$, using another I/Q modulator, and passed through an optical delay line which gives 3166 symbols delay, to de-correlate the channels of the shifted spectrum from the original. After amplification, the shifted spectrum was combined with the original path through another coupler. A PDM emulator with 371 symbols de-correlation delay was then used to form a 16-band PDM signal. The $1 \mathrm{GHz}$ guard band in this experiment is used to allow for frequency drift of the ECLs, which was dominated by the mechanical backlash in their frequency setting dials.

The PDM signals were then transmitted through a fiber recirculating loop, comprising two $80-\mathrm{km}$ spans of standard single mode fiber $(0.19 \mathrm{~dB} / \mathrm{km}$ loss and $16-18 \mathrm{ps} /(\mathrm{nm} * \mathrm{~km})$ $\mathrm{CD}$ parameter), a WS for gain flattening and three erbium 

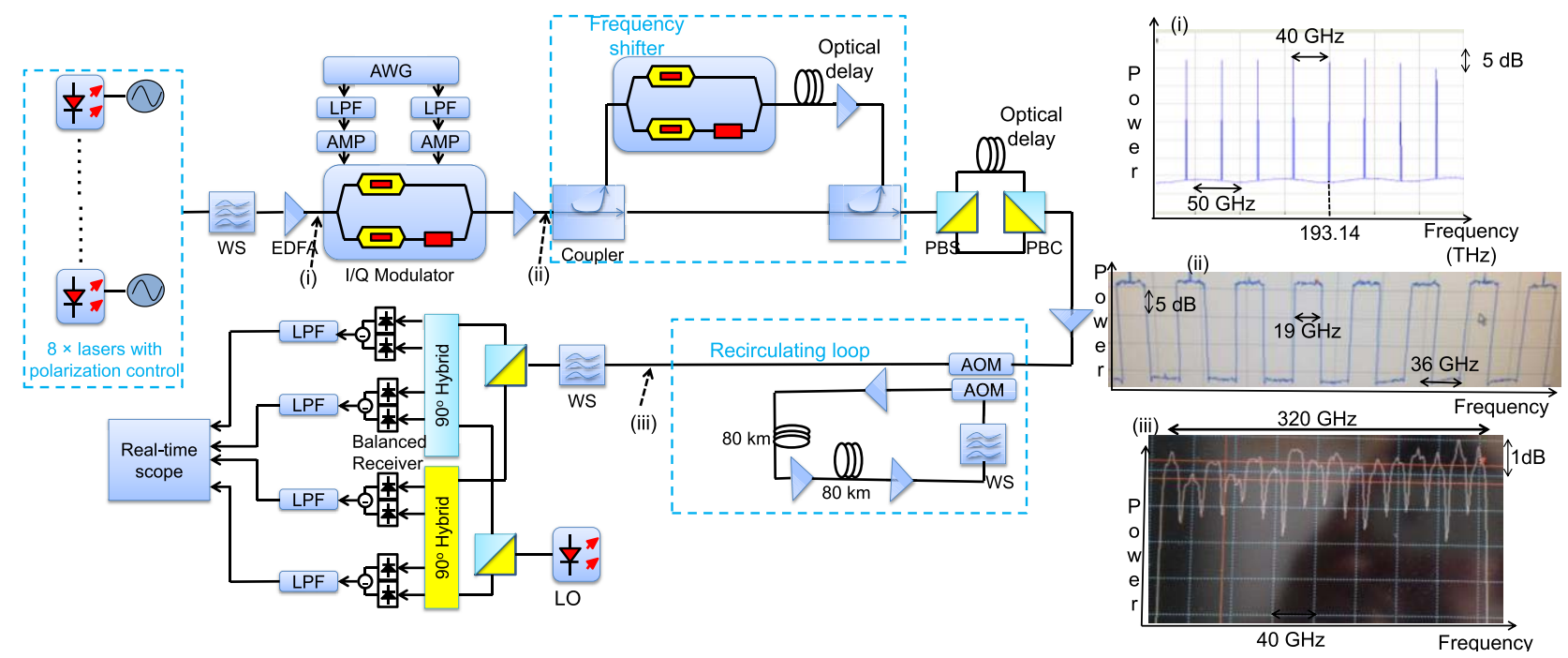

Fig. 3. Experimental setup.

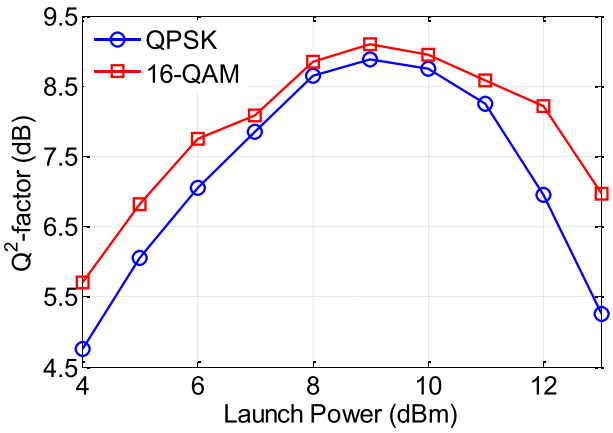

Fig. 4. Measured $Q^{2}$-factors versus different total launch power.

doped fiber amplifiers (EDFAs) with about $5 \mathrm{~dB}$ noise figure each. The power loss due to the loop equipment (WS, acoustooptic modulator (AOM), and coupler) is around $16 \mathrm{~dB}$. The signals were transmitted over 30 loops $(4800 \mathrm{~km})$ and 5 loops $(800 \mathrm{~km})$ for QPSK and 16-QAM, respectively. The $320 \mathrm{GHz}$ wide, 16-channel super-channel signal spectrum after transmission is shown as inset (iii) of Fig. 3, the power difference between received channels are within $0.5 \mathrm{~dB}$. At the receiver, the signal was first filtered by another WS to suppress out-of-band noise and then fed into a coherent receiver. Finally the RF signals were captured by a 4-channel 40-GSa/s digital oscilloscope with $16 \mathrm{GHz}$ electrical bandwidth for offline processing.

The offline DSP processing follows the scheme discussed in Section II. Fig. 4 shows the measured $\mathrm{Q}^{2}$-factor $\left(Q^{2}(d B)=\right.$ $\left.20 \log _{10}\left(\sqrt{2} e r f c^{-1}(2 B E R)\right)\right)$ versus different launch powers for the $9^{\text {th }}$ channel for QPSK and 16-QAM after $800-\mathrm{km}$ and $4800-\mathrm{km}$ transmission, respectively. It is clear that $9 \mathrm{dBm}$ is the optimal launch power. We stick to the optimal launch power for the rest of the measurements. Figure 5 shows the performance of the $9^{\text {th }}$ channel with different numbers of blind equalizer taps for three scenarios: our proposed blindonly equalizer; the reference ( $\mathrm{RRC}+$ blind) with FOC performed prior to matched filtering $(\mathrm{RRC}+\mathrm{Blind}$ pre-FOC); and (RRC + blind) with FOC performed after blind equalization
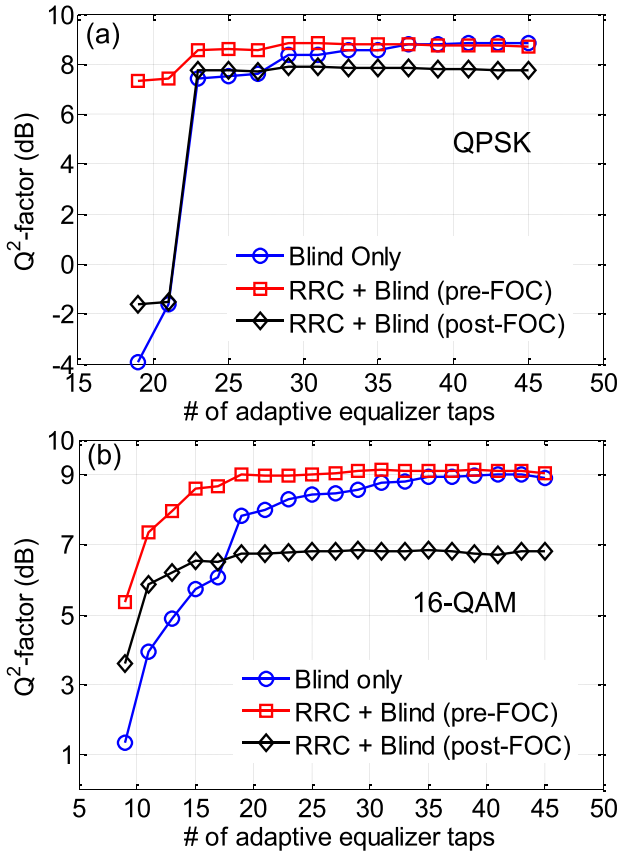

Fig. 5. $\mathrm{Q}^{2}$-factors versus number of blind equalizer taps for three equalization schemes for: (a) QPSK after 4800-km and (b) 16-QAM after 800-km.

(RRC + Blind post-FOC), to show the sensitivity to frequency offset.

If the frequency offset is compensated before the matched filter (using spectrum method [17] with a large number of signal samples to achieve accurate frequency offset estimation), RRC + Blind can reach optimal performance with 23 and 17 blind equalizer taps for QPSK and 16-QAM, respectively; however, if RRC filtering is applied without FOC, there are about $1 \mathrm{~dB}$ and $2.5 \mathrm{~dB} \mathrm{Q}^{2}$-factor penalties for the QPSK and 16-QAM systems, respectively no matter how many taps are used. In Fig. 5, the actual frequency offsets for QPSK and 16-QAM were about $240 \mathrm{MHz}$ and $280 \mathrm{MHz}$, respectively With the same frequency offset, our preliminary simulations predict a best case $\mathrm{Q}^{2}$-factor penalty of close to 0.8 and $1.5 \mathrm{~dB}$ for QPSK and 16QAM, respectively. The required number of 

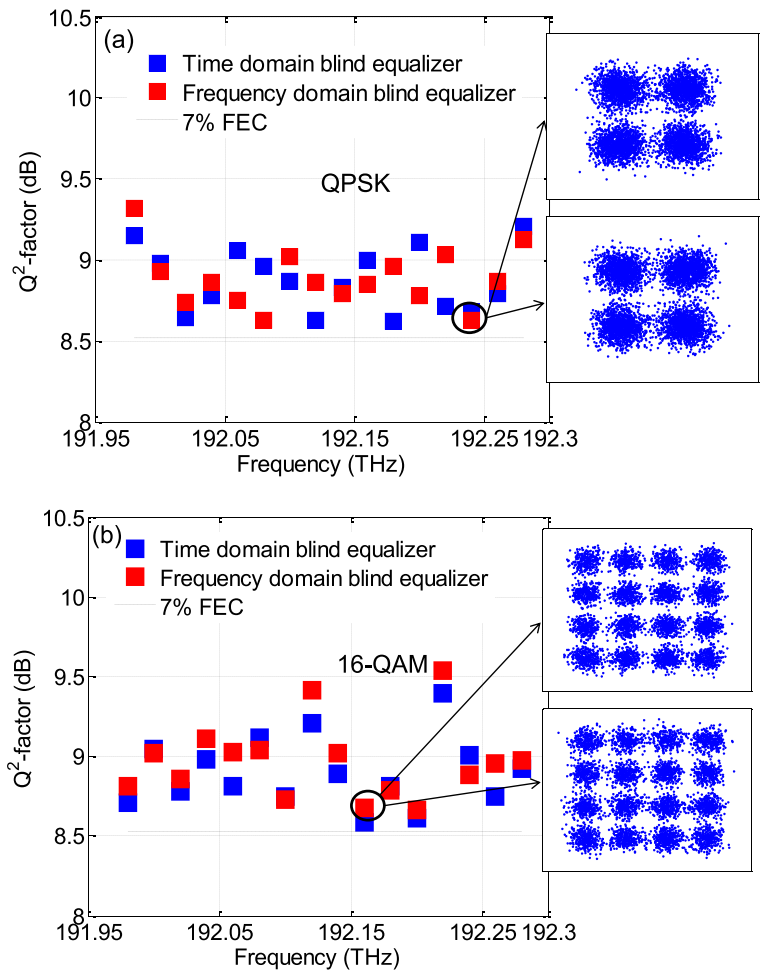

Fig. 6. $Q^{2}$-factors for all 16 bands using a single time domain and frequency domain blind equalizer without matched filtering for: (a) QPSK after 4800-km and (b) 16-QAM after $800-\mathrm{km}$

blind equalizer taps for QPSK transmission is higher than 16-QAM due to the larger residual $\mathrm{CD}$, caused by longer transmission distance and the fixed mismatch between the estimated CD parameter within the $\mathrm{CD}$ equalizer and the true $\mathrm{CD}$ of the fiber. By comparing Fig. 5a and b, 16-QAM appears more sensitive to frequency offset penalty.

When a single blind equalizer is used, it requires 33 and 41 taps (10 and 25 taps longer than RRC + blind) for the filter to converge to the optimal performance for the QPSK and 16-QAM systems, respectively. In this case, according to Fig.2, using a single frequency domain blind equalizer with 64 taps (82 comp.multi/sym) is much more computationally efficient than single time domain equalizer with 33/41 taps (200/248 comp.multi/sym) and RRC + blind (94 comp.multi/sym), and is also insensitive to frequency offset.

We implemented the 64-tap frequency domain blind equalizer and compared its performance to the 31/41-tap time domain FIR adaptive equalizer for QPSK and 16-QAM systems, respectively. As shown in Fig. 6 it is clear that the performance for all 16 bands is similar for both of these filter implementations. This shows conclusively that the frequency domain blind equalizer is as effective as the RRC + blind scheme, with the benefit of being more computationally efficient and insensitive to frequency offset. The performance for all bands are above the 7\% FEC threshold $8.52 \mathrm{~dB}$ $\mathrm{Q}^{2}\left(\mathrm{BER}=3.8 \times 10^{-3}\right)$.

\section{CONCLUSION}

We have investigated the design of matched filtering and blind equalization in a Nyquist-WDM system with a very small $(\alpha=0.01)$ roll-off factor. It is demonstrated that implementing separate RRC filtering causes sensitivity to frequency offset-it is better to combine this with the equalizer, as the equalizer will effectively adapt the matched filter to account for frequency offsets. A small increase in the number of taps of the equalizer is all that is required. Furthermore, implementing this longer blind equalizer in frequency domain is actually more efficient than implementing the RRC and the blind equalizers separately, because frequency-domain implementations give the best benefit for longer filters. We have implemented the blind equalizer experimentally using $1.14 \mathrm{~Tb} / \mathrm{s}$ Nyquist PDM-QPSK over $4800-\mathrm{km}$ and $2.27 \mathrm{~Tb} / \mathrm{s}$ Nyquist PDM-16-QAM over 800-km including 7\% FEC, proving that a single long-tap frequency domain blind equalizer achieves the optimal performance at minimum computational cost.

\section{REFERENCES}

[1] P. Winzer, "Beyond 100 G Ethernet," IEEE Commun. Mag., vol. 48, no. 7, pp. 26-30, Jul. 2010.

[2] M. Paskov, D. Lavery, and S. J. Savory, "Blind equalization of receiver in-phase/quadrature skew in the presence of Nyquist filtering," IEEE Photon. Technol. Lett., vol. 25, no. 24, pp. 2446-2449, Dec. 15, 2013.

[3] J. X. Cai et al., " $112 \times 112 \mathrm{~Gb} / \mathrm{s}$ transmission over $9,360 \mathrm{~km}$ with channel spacing set to the baud rate (360\% spectral efficiency)," in Proc. 36th Eur. Conf. Exhibit. Opt. Commun., Torino, Italy, Sep. 2010, pp. 1-3.

[4] X. Zhou et al., "High spectral efficiency $400 \mathrm{~Gb} / \mathrm{s}$ transmission using PDM time-domain hybrid 32-64 QAM and training-assisted carrier recovery," J. Lightw. Technol., vol. 31, no. 7, pp. 999-1005, Apr. 1, 2013.

[5] C. Zhu et al., "1.15 Tb/s Nyquist PDM 16-QAM transmission with joint matched filtering and frequency-domain equalization," in Proc. Opt. Fiber Commun. Conf., San Francisco, CA, USA, 2014, paper Th4D.4.

[6] B. Châtelain et al., "A family of Nyquist pulses for coherent optical communications," Opt. Exp., vol. 20, no. 8, pp. 8397-8416, Apr. 2012.

[7] J. G. Proakis, Digital Communications, 4th ed. New York, NY, USA: Mcgraw-Hill, 2001.

[8] J. Wang, C. Xie, and Z. Pan, "Matched filter design for RRC spectrally shaped Nyquist-WDM systems," IEEE Photon. Technol. Lett., vol. 25, no. 23, pp. 2263-2266, Dec. 1, 2013.

[9] C. Zhu et al., "Experimental investigation of matched filtering and blind adaptive equalizer design for Nyquist-WDM systems," in Proc. Optoelectron. Commun. Conf., Melbourne, Australia, 2014, paper MO2B-5.

[10] S. J. Savory, "Digital coherent optical receivers: Algorithms and subsystems," IEEE J. Sel. Topic Quantum Electron., vol. 16, no. 5, pp. 1164 1179, Sep./Oct. 2010.

[11] R. Kudo et al., "Coherent optical single carrier transmission using overlap frequency domain equalization for long-haul optical systems," J. Lightw. Technol., vol. 27, no. 16, pp. 3721-3728, Aug. 15, 2009.

[12] C. Zhu et al., "Frequency-domain blind equalization for long-haul coherent pol-mux 16-QAM system with CD prediction and dual-mode adaptive algorithm," IEEE Photon. J., vol. 4, no. 5, pp. 1654-1661, Oct. 2012.

[13] P. J. Winzer, A. H. Gnauck, C. R. Doerr, M. Magarini, and L. L. Buhl, "Spectrally efficient long-haul optical networking using 112-Gb/s polarization-multiplexed 16-QAM," J. Lightw. Technol., vol. 28, no. 4, pp. 547-556, Feb. 15, 2010.

[14] E. Ip and J. M. Kahn, "Feedforward carrier recovery for coherent optical communications," J. Lightw. Technol., vol. 25, no. 9, pp. 2675-2692, Sep. 1, 2010.

[15] C. Zhu, A. V. Tran, F. N. Hauske, S. Chen, T. Anderson, and E. Skafidas, "Low-complexity fractionally-spaced frequency domain equalization with improved channel estimation for long-haul coherent optical systems," in Proc. Opt. Fiber Commun. Conf. Expo. Nat. Fiber Opt. Eng. Conf. (OFC/NFOEC), Anaheim, CA, USA, Mar. 2013, pp. 1-3.

[16] M. S. Faruk and K. Kikuchi, "Adaptive frequency-domain equalization in digital coherent receivers," Opt. Exp., vol. 19, no. 13, pp. 12789-12798, Jun. 2011.

[17] K. Piyawanno, M. Kuschnerov, and B. Lankl, "Fast and accurate automatic frequency control for coherent receivers," in Proc. Eur. Conf. Opt. Commun., Vienna, Austria, 2009, p. 7.3.1. 